\title{
CLOUD TOP HEIGHT ESTIMATION FROM WRF MODEL: APPLICATION TO THE INFRARED CAMERA ONBOARD EUSO-BALLOON
}

\author{
A. Merino; J.L. Sánchez; L. López; E. Gascón; E. García-Ortega; L. Hermida; \\ Grupo de Física de la Atmósfera. University of León, Spain. \\ E-mail: amers@unileon.es \\ S. Fernández-González \\ Department of Astrophysics and Atmospheric Sciences, Faculty of Physical Sciences. UCM, \\ Madrid, Spain
}

\section{J.F. Soriano; G. Sáez-Cano; L. Del Peral}

Space and Astroparticle Group. UAH, Madrid, Spain

M. Reyes; E. Joven; Y. Martín; J. Licandro

Instituto de Astrofísica de Canarias (IAC), Vía Lactea S/N, Tenerife, Spain.

\section{S. Franchini}

IDR/UPM, Universidad Politécnica de Madrid. Madrid, Spain

\section{D. Rodríguez Frías*}

SPace \& AStroparticle (SPAS) Group, UAH, Madrid, Spain.

IFIC, CSIC, Universitat de València

Dpto. Física Atómica, Molecular y Nuclear, Universitat de València.

ISDC, Astronomy Dept. University of Geneva, Switzerland.

\section{for the JEM-EUSO Collaboration}

EUSO-BALLOON was launched on August 24, 2014 from Timmins (Canada) with a bispectral Infrared Camera onboard intended to measure the cloud coverage during the flight. Clouds at mid and upper levels of the Troposphere are crucial for a proper reconstruction of the main parameters of the Ultra-High Energy Cosmic Rays (UHECR).Therefore, determining Cloud Top Height $(\mathrm{CTH})$ with high accuracy is crucial to estimate the effect of clouds on these measurements. With this aim, we have developed a method to extract CTH parameter via vertical profiles predicted by the Weather Research Forecast (WRF) model. Moreover, we have evaluated model ability to represent temperature and humidity profiles in different climatic regions of the globe.

The 34th International Cosmic Ray Conference,

30 July- 6 August, 2015

The Hague, The Netherlands

\footnotetext{
* Speaker.
} 


\section{Introduction}

The arrival of Ultra-High Energy Cosmic Rays (UHECRs) in the Earth's atmosphere causes Extensive Air Showers (EAS) that produce ultraviolet radiation, which is detected and measured by the telescope from the EUSO programme ([12]). However, atmospheric conditions, and especially the presence of clouds, are known to introduce high rates of uncertainty into UV radiation measurements. Accuracy in determining EAS parameters, such as the energy of the primary particle or the shower maximum, is strongly dependent on the atmospheric conditions (such as temperature, pressure or humidity) at the moment when the events take place. These parameters may alter the development and detection of EAS. Unlike ground-based telescopes, JEM-EUSO will be able to observe the majority of the shower development even in the presence of certain types of clouds (especially when the clouds are optically thin or when their Cloud Top Height (CTH) is located below the shower maximum). As the telescope will monitor different atmospheric conditions at the same time, a precise knowledge of the spatial atmospheric properties (mainly cloud coverage and cloud top height) inside the telescope Field of View (FoV) is mandatory in order to correctly reconstruct the cosmic ray particle properties. In order to know the atmospheric conditions and properties of the clouds in the FoV of the telescope, the JEM-EUSO Space Observatory is implementing an Atmospheric Monitoring System (AMS) that will include a bi-spectral IR-Camera ([11]; [13]) and a LIDAR. For retrieved CTH using remote sensors, it must be recalled that thermal emission of the cloud comes from its uppermost layer. Thus, the retrieved CTH is not the physical cloud boundary but the radiatively effective one [7]. In addition, when the temperature profile includes thermal inversions, the conversion between brightness temperature and CTH results in greater uncertainties, which produce large errors. These limitations are especially relevant for optically thin clouds, which produce strong uncertainties in the extraction of cloud properties. Advances in computing technology have enabled a substantial capacity for Numerical Weather Prediction (NWP) models to assimilate observations, thereby improving the skill of meteorological predictions [1]. Currently, the improved vertical and horizontal resolutions of such models permits cloud features to be simulated with great precision. Thus, NWP models have been evaluated for calculating CTH of low clouds, because of their independence of disturbances from higher clouds. As mentioned above, algorithms based on remote sensing have greater uncertainties for multilayer cloud events.

Here the aim is to develop a method to extract CTH using vertical profiles predicted by the Weather Research and Forecasting model (WRF; [14]) together with Brightness Temperatures (BT) of the IR-Camera. The same concept of using WRF model is being used in ([15]) where a different analysis is carried out and the results compared with the very accurate spectroradiometer MODIS, however in this study we have developed a new algorithm to retrieve CTH starting from BT of IR-Camera. Thus, we have obtained the first CTH product in EUSO-BALLOON images, that is a mandatory product in order to correctly reconstruct the cosmic ray particle properties.

\section{Methodology}

First, we evaluated the ability of the model to represent temperature and humidity profiles in different climatic regions of the globe (Figure 1). Obtaining good temperature and humidity profiles is essential for subsequent algorithm development. With this goal in mind, we established 


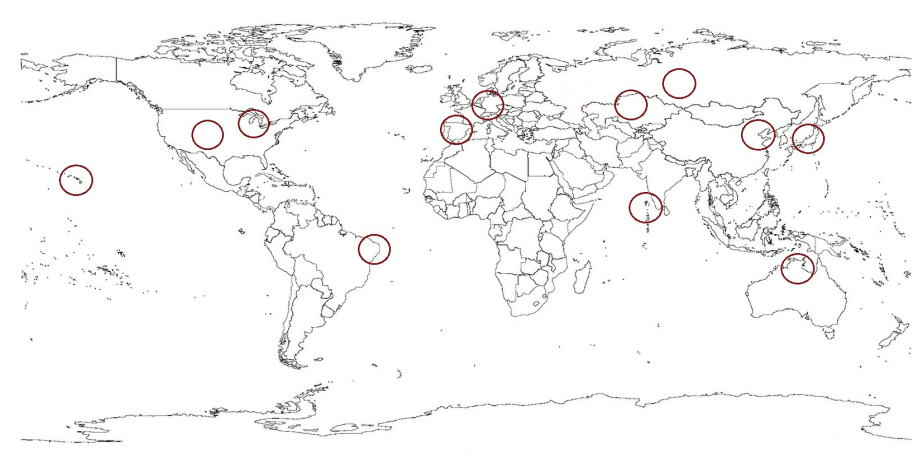

Figure 1: The red circles show the areas where the profiles from the WRF model were validated with radiosondes.

\begin{tabular}{|c|c|c|}
\hline & Station 1 & Station 2 \\
\hline \multirow{2}{*}{ Europe } & Madrid-08221 & Zaragoza-08160 \\
\cline { 2 - 3 } & Meiniagen-10548 & Praha Libus-11520 \\
\hline \multirow{2}{*}{ Sorth America } & Denver-72469 & Grand Junction-72476 \\
\cline { 2 - 3 } & Moosonee-71836 & Gaylord-72634 \\
\hline \multirow{2}{*}{ Southeast Asia } & Natal-82599 & Fernando de Noronha-82400 \\
\cline { 2 - 3 } & Kagosima-47827 & Fukusoka-47807 \\
\cline { 2 - 3 } Mideast & Beijing-54511 & Hohhot-53463 \\
\hline Artic & Turuhansk-34122 & Kalakh-34247 \\
\hline Australia & Darwin-94120 & Bor-23884 \\
\hline Pacific & PHLI Lihue-91165 & Gove-94150 \\
\hline Indian & Amini Divi-43311 & Minicoy-43369 \\
\hline
\end{tabular}

Table 1: Radiosonde stations selected in each study window.

12 windows covering specific parts of the Earth all the year round. From these, points with available soundings were selected to calibrate and obtain the optimal WRF configuration for producing vertical temperature profiles. Within the WRF, we defined two domains for each window, with spatial resolutions 15 and $5 \mathrm{~km}$. Vertical resolution was established with 58 levels, as we required highly accurate profiles. The initial and profile conditions were extracted from NCEP (National Centers for Environmental Prediction) re-analysis with $1^{\circ}$ of resolution. In each study window, we evaluated a series of parametrizations (microphysics, cumulus, radiation). To do so, we compared vertical profiles obtained by the WRF for each parametrization, using seasonal sounding data in each study area. In each study window, a total of 20 simulations were carried out with the WRF model for all of the seasons of the year. For each simulation, data were taken from two radiosonde stations in domain two (5 km spatial resolution), together with the temperature and humidity profiles from the WRF model in the same locations and at the same times as the radiosonde stations. As a result, 40 radiosonde vs. WRF comparisons were obtained in each of the study windows. Table 1 shows the stations from which the radiosonde data were obtained. 

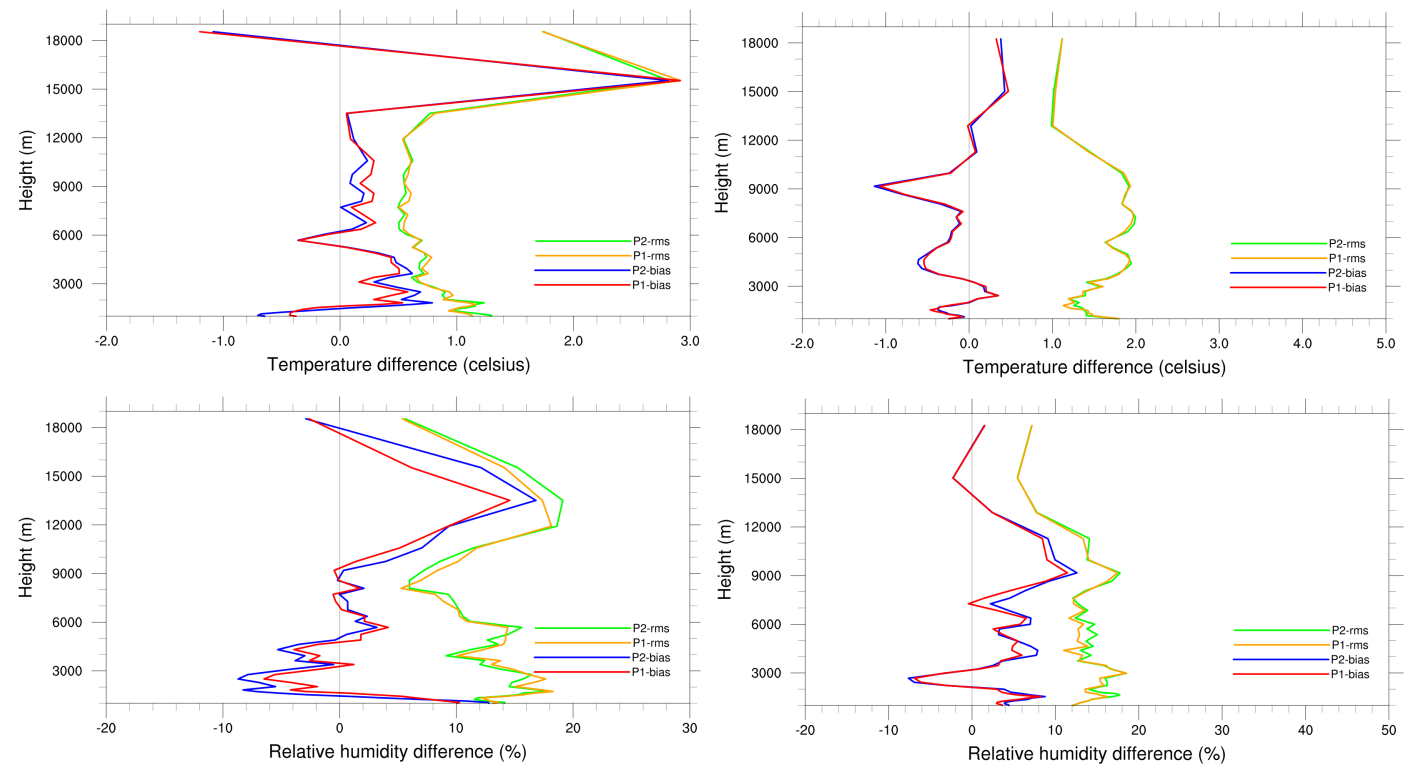

Figure 2: Comparison of WRF/radiosonde vertical profiles: average bias and rms of 40 profiles. Left: Brazil. Right: Canada. P1 and P2 represent Goddard and Thompson schemes, respectively.

Once we obtained the optimum physical configuration of the model for each climatic region, we developed an application to automatically determine cloud top height at each pixel of the infrared camera images, taking as input BT plus the temperature and humidity profiles output from the WRF at each pixel. As a result, in each cloud pixel from the IR-Camera, the BTs were considered as the cloud top temperature and transformed in $\mathrm{CTH}$ using the closest vertical temperature and humidity profile obtained by the WRF model. A pixel is considered cloudy when BT at 10.8 micron is less than the surface temperature retrieved by WRF. In these pixels CTH is computed in two parts. The first one considering clouds as black body, so BT at 10.8 microns is regarded as cloud top temperature. Using WRF vertical profiles of temperature CTH is retrieved from cloud top temperature. The heights so calculated are less than actual height for distant cloud of black body. So, to correct this effect, in the second part, the height computed previously is raised depending on the atmospheric conditions simulated by WRF fields: relative humidity profiles, ice water drop profiles and liquid water drop profiles. These fields, involved with moisture conditions, allow to know the layers where clouds are identified by WRF.

\section{Results}

High spectral resolution IR sounding based in satellite measurements are a principal source of atmospheric water vapor and temperature data in areas where few conventional observations are made. Also, high-resolution numerical models such as WRF are another alternative way of obtaining temperature and humidity data to support the limited temporal resolution of polar satellite 

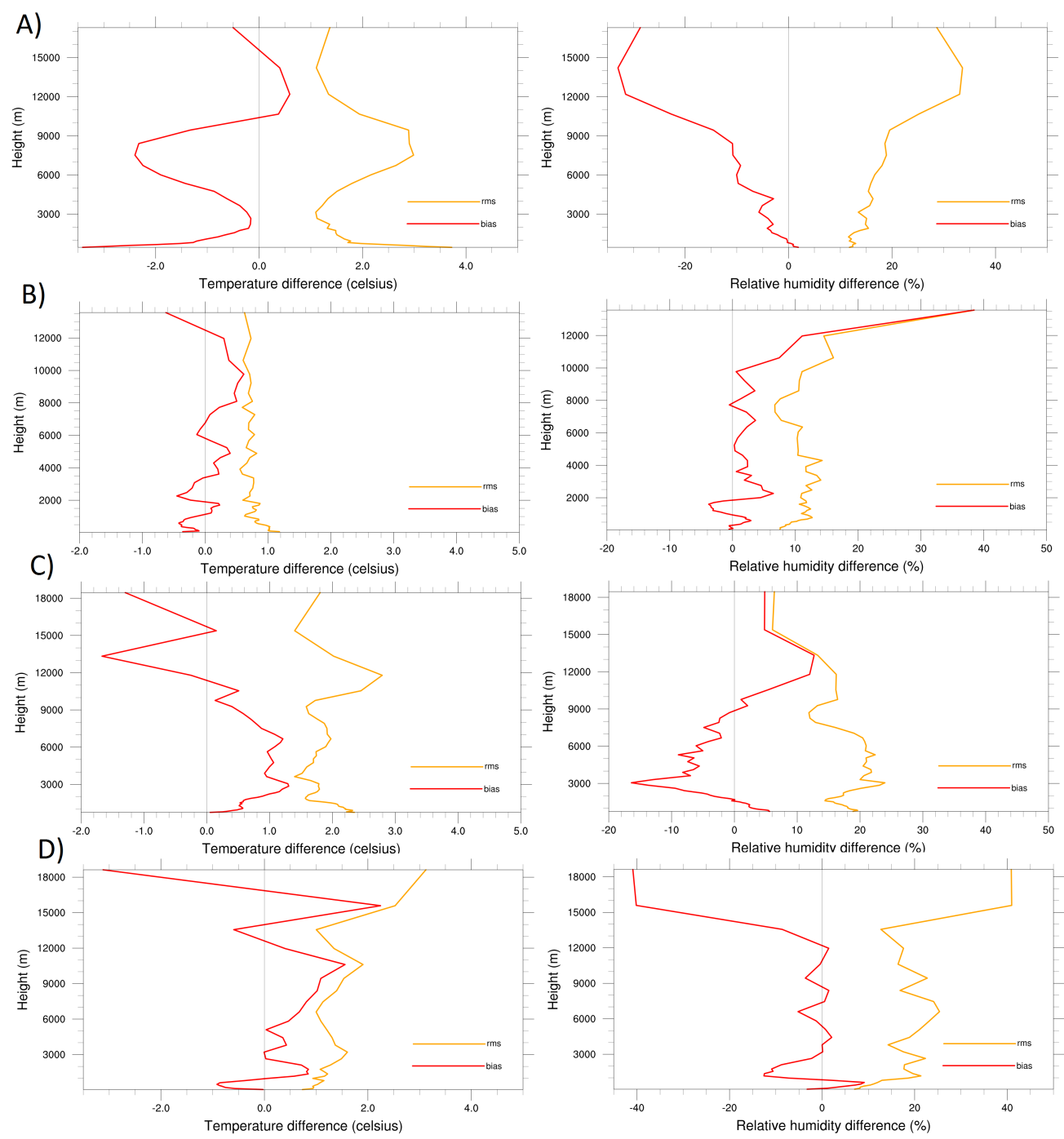

Figure 3: Comparison of WRF/radiosonde vertical profiles: average bias and RMS of 40 profiles. A) Arctic. B) Australia. C) Europe. D) India.

data. Obtaining high precision vertical temperature and humidity profiles is essential for numerical models to correctly characterize the cloud structures present in the data, as they allow the analysis of the vertical cloud structure ([8]). Different studies have evaluated the ability of numerical weather prediction models to simulate the temperature and humidity in the lower troposphere using radiosondes ([2]; [6]). In this study, the vertical temperature and humidity profiles were evaluated using two radiosondes from each study area, taken over 20 simulations of 36 hours during different seasons of the year. The evaluation was carried out by taking the closest WRF grid point to each radiosonde. In all of the study windows, the Kain-Fritsch cumulus scheme parametrization was used ([9]) because an evaluation had already been carried out in previous studies ([5]). In the case of the moisture scheme, two different parametrizations were evaluated: the Goddard Scheme (P1; [17]; [16]) and Thompson (P2; [18]) for Canada and Brazil, in order to test the influence of the parametrization of humidity in an equatorial zone, and another corresponding to mid-latitudes. 
In the case of the other parametrizations, the Dudhia Shortwave Scheme ([3]) and The Noah land surface model ([4]) were applied for the short wave and long wave option.

Figure 2 shows the mean result of validating the temperature and humidity profiles over the 20-day study period and for the two radiosonde stations selected in each study area. We can see that in this case, the humidity parametrization does not significantly influence the results, as the difference in terms of temperature and relative humidity is minimum, and for this reason it was decided to choose the Goddard Scheme for the other windows. Regarding the verification of the profiles, in the case of Brazil an RMS value can be seen around one degree Celsius in the mid and low troposphere, a difference that increases to 3 degrees in the tropopause. In the case of Canada, the differences increased up to 2 degrees, although there is an improvement in the proximity of the tropopause. In the case of the relative humidity profiles, the behavior is similar in both areas, with RMS value of between $10-20 \%$.

The verification of the vertical temperature and relative humidity profiles of the WRF model with the established configuration for the different windows is shown in figures 3 . In general, it can be observed that the profiles for the locations closest to the equator have the best fit. It can also be seen that the RMS value in the window for Australia and India is around one degree Celsius in the temperature profiles and between $10-20 \%$ in the humidity profiles in low and middle layers of the troposphere. On the contrary, as we move towards higher latitudes, the profiles do not fit so well, with the window located in the Arctic having an RMS value of 2-3 degrees for the temperature, and between $20-30 \%$ for the humidity. One possible cause of these results is the spatial variability of the temperature and humidity in different parts of the planet. In tropical zones, the temperature and humidity gradients are usually much lower than in middle-high latitudes. In high latitudes, where the polar front is located, small errors in the location made by the model for these boundary zones can cause major errors in the comparisons of the profiles. This can also be seen in maritime zones, where deviations are also lower than in continental zones that are far from oceans.

Once the temperature and humidity profiles were verified, we have developed an algorithm to compute CTH using as input the Brightness temperature of IR-Cam and WRF outputs (see methodology section). As example here one scene of EUSO-BALLOON is shown in figure 4. In this figure we can see cloud top height between 4-7 km, considering cloud as black body (Figure4-Left), raising until $10 \mathrm{~km}$ when WRF correction is applied (Figure4-right). The preliminary validation of the algorithm was performed with the CALIOP LIDAR, showing good agreement particularly for high cloud tops, obtaining a RMS of 2.0-0.5 km for different cloud scenes.

\section{Conclusions}

It was found that the parametrization of humidity in the WRF model does not significantly influence the temperature and humidity profiles, as these variables have an explicit resolution from the dynamics of the model. The verifications made for the temperature and humidity profiles of the WRF model with radiosondes show RMS of around 1-2 degrees Celsius for the temperature and 10$30 \%$ for the humidity. These discrepancies were found to be higher in the mid-high latitudes than in tropical zones, and in the troposphere close to the tropopause. An algorithm is developed in two parts to retrieve CTH using as input the BT of the IR-Cam and WRF outputs. Preliminary validation with CALIOP LIDAR shows good agreement for high cloud tops, improving other algorithms 

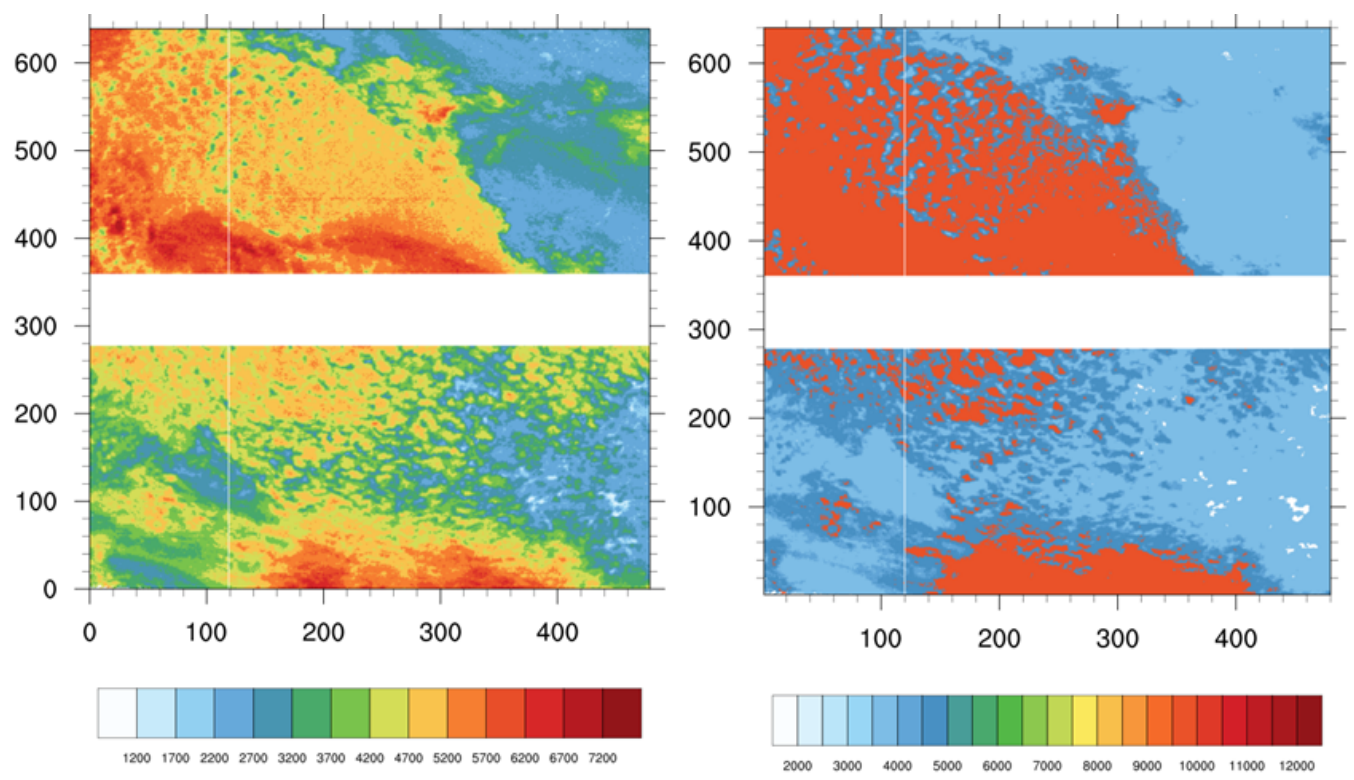

Figure 4: CTH of EUSO-BALLOON scene for 0739 UTC.Left: CTH of algorithm first part. Right: CTH final, including WRF corrections.

developed with multispectral radiometers ([10]). In the future, the numerical simulation techniques described here will be combined with information from the bi-spectral IR-Cam in the JEM-EUSO telescope. As a result, by using the advantages of the different spatial and temporal resolutions of each system, a cloud characterization will be made of the telescope's field of view, to correctly detect and measure the EAS.

Acknowledgment: This work is supported by the Spanish Government MICINN \& MINECO under projects AYA2009-06037-E/AYA, AYA-ESP 2010-19082, AYA2011-29489-C03-01 and AYA2011-29489-C03-02, AYA-ESP201239115-C03-01 and AYA-ESP2012-39115-C03-03, AYA-ESP 2013-47816-C4, MINECO/FEDER-UNAH13-4E-2741, CSD2009-00064 (Consolider MULTIDARK) and by Comunidad de Madrid under projects S2009/ESP-1496 \& S2013/ICE2822. The calculations were performed using the Space and Astroparticle SPAS-UAH Cluster. M. D. Rodríguez Frías acknowledges the Swiss National Science Foundation (SNSF) for a Sabbatical research stay at the University of Geneva. L. del Peral acknowledges a senior grant for a Sabbatical stay at University of Geneva from the Spanish Ministerio de Educación, Cultura y Deporte under the "Salvador de Madariaga Programa Estatal de Promoción del Talento y su Empleabilidad en I+D+i, Subprograma Estatal de Movilidad del Plan Estatal de Investigación Científica y Técnica y de Innovación 2013-2016" M D Rodriguez Frias acknowledges a grant under the "Atraccio de Talent" program from the Vicerrectorado de Investigación de la Universidad de Valencia (Spain).

\section{References}

[1] Ali, A. H., 2004. Application of neural principal components to climate data. J. Atmos. Oceanic Technol., 21, 149-158.

[2] Bromwich, D. H., Cassano, J., Klein, T., Heinemann, G., Hines, K., Steffen, K., Box, J., 2000. Mesoscale modeling of katabatic winds over Greenland with the polar MM5. Mon. Wea. Rev., 129, 2290-2309.

[3] Dudhia, J., 1989. Numerical study of convection observed during the winter monsoon experiment using a mesoscale two-dimensional model, J. Atmos. Sci., 46, 3077-3107. 
[4] Chen, F. and J.Dudhia, 2001. Coupling an Advanced Land Surface-Hydrology Model with the Penn State-NCAR MM5 Modeling System. Part I: Model Implementation and Sensitivity. Mon. Wea. Rev., 129, 569-585.

[5] García-Ortega, E., López, L., Sánchez, J.L., 2001. Atmospheric patterns associated with hailstorm days in the Ebro Valley, Spain. Atmos. Res., 100 (4), 401-427.

[6] Guo, Z., Bromwich, D., Cassano, J., 2003. Evaluation of polar MM5 simulations of Antarctic atmospheric circulation. Mon. Wea. Rev., 131, 384-411.

[7] Hamann, U., Walther, A., Baum, B., Bennartz, R., Bugliaro, L., Derrien, M., Francis, P.N., Heidinger, A., Joro, S., Kniffka, A., Le Gléau, H., Lockhoff, M., Lutz, H.-J., Meirink, J.F., Minnis, P., Palikonda, R., Roebeling, R., Thoss, A., Platnick, S., Watts, P., Wind, G., 2014. Remote sensing of cloud top pressure/height from SEVIRI: Analysis of ten current retrieval algorithms. Atmospheric Measurement Techniques, 7 (9), pp. 2839-2867.

[8] Jin, X., Hanesiak, J., Barber, D., 2007. Detecting cloud vertical structures from radiosondes and MODIS over Arctic first-year sea ice. Atmos. Res., 83 (1), 64-76.

[9] Kain, J.S., 2002. The Kain-Fritsch convective parameterization: an update. J. Appl. Meteorol. 43 (1), 170-181.

[10] Menzel, W.P., Frey, R.A., Baum, B.A., 2013. Cloud top properties and cloud phase algorithm theoretical basis document. Collection 6, Version 10, University of Wisconsin-Madison.

[11] Neronov, A., Rodriguez-Frias, M. D., Toscano, S. and Wada, S., 2013. Atmospheric Monitoring System of the JEM-EUSO telescope, Proc. 33rd Int.Cosm. Ray Conf., 91-94, Rio de Janeiro.

[12] Piccoza, P. et al. 2015. The JEM-EUSO program, Proc. 34rd Int.Cosm. Ray Conf., Hague.

[13] Rodriguez-Frias, M. D., Licandro, J., Sabau, M. D., Reyes, M., Belenguer,T., Gonzalez-Alvarado,M.C., Joven, E., Morales de los Rios, J. A., Saez-Palomino, M., Prieto, Saez-Cano, G., Carretero, J., Perez-Cano, S., del Peral, L., 2013. Towards the preliminary Design Review of the Infrared Camera of the JEM-EUSO Collaboration. Proc. 33rd Int. Cosm. Ray Conf., 95-98, Rio de Janeiro.

[14] Skamarock, W.C., Klemp, J.B., Dudhia, J., Gill, D.O., Barker, D.M., Wang, W., Powers, J.G., 2005. A Description of the Advanced Research WRF Version 2. NCAR Tech. Note NCAR/TN-4681STR (88 pp.).

[15] Tabone, I. et al., 2015. The WRF model contribution to the Cloud Top Height retrieval in EUSO-Balloon experiment, Proc. 34rd Int.Cosm. Ray Conf., Hague.

[16] Tao, W.-K., Simpson, J., 1993. Goddard Cumulus Ensemble Model. Part I: Model Description. Terr. Atmos. Ocean. Sci. 4, 3572.

[17] Tao, W.K., Simpson, J., McCumber, M., 1989. An ice-water saturation adjustment. Mon. Weather Rev. $117,231-235$.

[18] Thompson, G. Paul R. Field, Roy M. Rasmussen, and William D. Hall, 2008: Explicit Forecasts of Winter Precipitation Using an Improved Bulk Microphysics Scheme. Part II: Implementation of a New Snow Parameterization. Mon. Wea. Rev., 136, 5095-5115. 